\title{
Extended Lorentz Cones and Variational Inequalities on Cylinders
}

\author{
Sándor Zoltán Németh ${ }^{1}$ • Guohan Zhang ${ }^{1}$
}

Received: 26 May 2015 / Accepted: 22 October 2015 / Published online: 6 November 2015

(C) The Author(s) 2015. This article is published with open access at Springerlink.com

\begin{abstract}
Solutions of a variational inequality problem defined by a closed and convex set and a mapping are found by imposing conditions for the monotone convergence with respect to a cone of the Picard iteration corresponding to the composition of the projection onto the defining closed and convex set and the difference in the identity mapping and the defining mapping. One of these conditions is the isotonicity of the projection onto the defining closed and convex set. If the closed and convex set is a cylinder and the cone is an extented Lorentz cone, then this condition can be dropped because it is automatically satisfied. In this case, a large class of affine mappings and cylinders which satisfy the conditions of monotone convergence above is presented. The obtained results are further specialized for unbounded box-constrained variational inequalities. In a particular case of a cylinder with a base being a cone, the variational inequality is reduced to a generalized mixed complementarity problem which has been already considered in Németh and Zhang (J Global Optim 62(3):443-457, 2015).
\end{abstract}

Keywords Isotone projections · Cones · Variational inequalities $\cdot$ Picard iteration · Fixed point

Mathematics Subject Classification $90 \mathrm{C} 33 \cdot 47 \mathrm{H} 07 \cdot 47 \mathrm{H} 99 \cdot 47 \mathrm{H} 09$

$凶$ Sándor Zoltán Németh s.nemeth@bham.ac.uk

Guohan Zhang gxz245@bham.ac.uk

1 School of Mathematics, University of Birmingham, Watson Building, Edgbaston, Birmingham B15 2TT, UK 


\section{Introduction}

In this paper, we will study the solvability of variational inequalities on closed and convex sets by using the isotonicity of the metric projection mapping onto these sets with respect to the partial order defined by a cone. Apparently, this approach has not been considered before.

Variational inequalities are models of various important problems in physics, engineering, economics, and other sciences. The classical Nash equilibrium concept can also be reformulated by using variational inequalities.

It is known (see Facchinei and Pang [1]) that a vector is a solution of a variational inequality defined by a closed and convex set and a mapping iff it is a fixed point of the composition of the projection onto the defining closed and convex set, and the difference in the identity mapping and the given mapping. If we could guarantee the isotonicity of the mappings in the latter composition with respect to the partial order defined by a cone, then we could construct an increasing sequence with respect to the partial order defined by the cone by using Picard's iteration. If we could also guarantee that this sequence is bounded from above with respect to the partial order defined by the cone, then this sequence would be convergent to a solution of the variational inequality (in fact, it would be convergent to a solution of the corresponding equivalent fixed point problem). It turns out that there is a class of variational inequalities and a class of cones that extend the Lorentz cone, for which this idea works very well. The only restriction is that the variational inequality has to be defined on a cylinder. Such problems appear in the practice. For example, the unbounded box-constrained variational inequalities are of this form. Based on the above idea, a theorem for finding solutions of variational inequalities on a cylinder will be presented, and an example will be given. Ideas similar to the above are presented in [2-7] for complementarity and implicit complementarity problems, but with a strong restriction on the cone defining the problem. The idea of monotone convergence for complementarity problems defined by general cones is considered first in Nemeth and Zhang [8]. The present paper extends the results of that paper for variational inequalities.

Several other papers [9-19] dealt with conditions of convergence for iterations of the above type by using similar conditions. However, neither of these works used the ordering defined by a cone for showing the convergence of the corresponding iterative scheme. Instead, they used as a tool the Banach fixed point theorem and assumed Kachurovskii-Minty-Browder-type monotonicity and global Lipschitz properties.

The structure of the paper is as follows: In the preliminaries, we introduce the terminology and notations used throughout this paper. In Sect. 3, we recall the definition and basic properties of the extended Lorentz cone, and describe all sets onto which the projection is isotone with respect to this cone. In Sect. 4, we will find solutions of a variational inequality by analyzing the monotone convergence with respect to a cone of the Picard iteration corresponding to the equivalent fixed point problem. In Sect. 5, we will specialize these results to variational inequalities defined on cylinders, by using the extended Lorentz cone for the corresponding monotone convergence above. In this case, we can drop the condition of Proposition 4.1 that the projection onto the closed and convex set in the definition of the variational inequality is isotone with respect to the extended Lorentz cone, because this condition is automatically satisfied, 
obtaining the more explicit result of Theorem 5.1. The latter result extends the results of Nemeth and Zhang [8] for mixed complementarity problems. In Sect. 6, a large class of affine mappings and cylinders which satisfy the conditions of Theorem 5.1 is presented. In Sect. 7, we further specialize the results for unbounded box-constrained variational inequalities.

\section{Preliminaries}

Denote by $\mathbb{N}$ the set of nonnegative integers. Let $k, m, p, q \in \mathbb{N} \backslash\{0\}$ and $\mathbb{R}^{m}$ be the $m$-dimensional real Euclidean vector space consisting of column vectors.

Identify the vectors of $\mathbb{R}^{m}$ by column vectors and consider the canonical inner product defined on $\mathbb{R}^{m}$ by $\mathbb{R}^{m} \times \mathbb{R}^{m} \ni(x, y) \mapsto\langle x, y\rangle:=x^{\top} y \in \mathbb{R}$ with induced norm $\mathbb{R}^{m} \ni x \mapsto\|x\|=\sqrt{\langle x, x\rangle} \in \mathbb{R}$.

The inner product in $\mathbb{R}^{p} \times \mathbb{R}^{q} \equiv \mathbb{R}^{p+q}$ is given by

$$
((x, u),(y, v)) \mapsto\langle x, y\rangle+\langle u, v\rangle .
$$

For simplicity, a closed set $L \subseteq \mathbb{R}^{m}$ will be called a cone iff $L \cap(-L)=\{0\}$ and $\lambda x+\mu y \in L$, whenever $\lambda, \mu \geq 0$ and $x, y \in L$, although in the subject literature often the term closed, convex, and pointed cone is used. Let $L \subseteq \mathbb{R}^{m}$ be a cone. Denote by $\leq_{L}$ the relation defined by $x \leq_{L} y \Longleftrightarrow y-x \in L$ and call it the partial order defined by $L$. The relation $\leq_{L}$ is reflexive, transitive, antisymmetric, and compatible with the linear structure of $\mathbb{R}^{m}$ in the sense that $x \leq_{L} y$ implies that $t x+z \leq_{L} t y+z$, for any $z \in \mathbb{R}^{m}$ and any $t \geq 0$. Moreover, $\leq_{L}$ is continuous at 0 in the sense that if $x^{n} \rightarrow x$ when $n \rightarrow \infty$ and $0 \leq_{L} x^{n}$ for any $n \in \mathbb{N}$, then $0 \leq_{L} x$.

For any closed and convex set $C$, denote by $P_{C}: \mathbb{R}^{m} \rightarrow \mathbb{R}^{m}$, the metric projection mapping onto $C$, that is, the mapping defined by $P_{C}(x) \in C$ and

$$
\left\|x-P_{C}(x)\right\|=\min \{\|x-y\|: y \in C\}, \forall x \in \mathbb{R}^{m} .
$$

Since $C$ is closed and convex, the projection mapping is well defined, and by its definition, it follows that

$$
P_{y+C}(x)=y+P_{C}(x-y), \forall x, y \in \mathbb{R}^{m} .
$$

It is known that $P_{C}$ is nonexpansive (see Zarantonello [20]), that is,

$$
\left\|P_{C}(x)-P_{C}(y)\right\| \leq\|x-y\|, \forall x, y \in \mathbb{R}^{m} .
$$

Let $L \subseteq \mathbb{R}^{m}$ be a cone. A mapping $F: \mathbb{R}^{m} \rightarrow \mathbb{R}^{m}$ is called L-isotone iff $x \leq_{L} y$ implies $F(x) \leq_{L} F(y)$.

The nonempty, closed, and convex set $C \subseteq \mathbb{R}^{m}$ is called $L$-isotone projection set iff $P_{C}$ is $L$-isotone.

The set $\Omega \subseteq \mathbb{R}^{m}$ is called $L$-bounded from below ( $L$-bounded from above) iff there exists a vector $y \in \mathbb{R}^{m}$ such that $y \leq_{L} x\left(x \leq_{L} y\right)$, for all $x \in \Omega$. In this case, $y$ is 
called a lower $L$-bound (upper L-bound) of $\Omega$. If $y \in \Omega$, then $y$ is called the $L$-least element ( $L$-greatest element) of $\Omega$.

Let $\mathcal{I} \subseteq \mathbb{N}$ be an unbounded set of nonnegative integers. The sequence $\left\{x^{n}\right\}_{n \in \mathcal{I}}$ is called $L$-increasing ( $L$-decreasing) iff $x^{n_{1}} \leq_{L} x^{n_{2}}\left(x^{n_{2}} \leq_{L} x^{n_{1}}\right)$, whenever $n_{1} \leq n_{2}$.

The sequence $\left\{x^{n}\right\}_{n \in \mathcal{I}}$ is called L-bounded from below ( $L$-bounded from above) iff the set $\left\{x^{n}: n \in \mathcal{I}\right\}$ is $L$-bounded from below ( $L$-bounded from above).

A cone $L$ is called regular iff any $L$-increasing sequence which is $L$-bounded from above is convergent (or equivalently, any $L$-decreasing sequence which is $L$-bounded from below is convergent). It is known (see McArthur [21]) that any cone in $\mathbb{R}^{m}$ is regular.

The dual of a cone $L \subseteq \mathbb{R}^{m}$ is defined by

$$
L^{*}=\left\{x \in \mathbb{R}^{m}:\langle x, y\rangle \geq 0, \forall y \in L\right\} .
$$

A cone $L$ is called subdual iff $L \subseteq L^{*}$ and self-dual iff $L=L^{*}$.

A cone $L \subseteq \mathbb{R}^{m}$ is called polyhedral iff it is generated by a finite number of vectors $v^{1}, \ldots, v^{k}$, that is,

$$
L=\operatorname{cone}\left\{v^{1}, \ldots, v^{k}\right\}:=\left\{\lambda_{1} v^{1}+\cdots+\lambda_{k} v^{k}: \lambda_{1}, \ldots, \lambda_{k} \geq 0\right\}
$$

The vectors $v^{1}, \ldots, v^{k}$ are called the generators of $L$.

The affine hyperplane with normal $u \in \mathbb{R}^{m} \backslash\{0\}$ and through $a \in \mathbb{R}^{m}$ is the set defined by

$$
\mathcal{H}(u, a)=\left\{x \in \mathbb{R}^{m}:\langle x-a, u\rangle=0\right\} .
$$

An affine hyperplane $\mathcal{H}(u, a)$ determines two closed halfspaces $\mathcal{H}_{-}(a, u)$ and $\mathcal{H}_{+}(u, a)$ of $\mathbb{R}^{m}$, defined by

$$
\mathcal{H}_{-}(u, a)=\left\{x \in \mathbb{R}^{m}:\langle x-a, u\rangle \leq 0\right\},
$$

and

$$
\mathcal{H}_{+}(u, a)=\left\{x \in \mathbb{R}^{m}:\langle x-a, u\rangle \geq 0\right\}
$$

\section{Extended Lorentz Cones}

Let $p, q$ be positive integers. For $a, b \in \mathbb{R}^{p}$ denote $a \geq b$ iff $b \leq_{\mathbb{R}_{+}^{p}} a$, that is, the components of $a$ are at least as large as the corresponding components of $b$. If the components of $a$ are larger than the corresponding components of $b$, then denote $a>b$. Denote by $e$ the vector in $\mathbb{R}^{p}$ with all components equal to one and by $e^{i}$ the canonical unit vectors of $\mathbb{R}^{p}$. In Nemeth and Zhang [8], we defined the following notion of an extended Lorentz cone:

$$
L(p, q)=\left\{(x, u) \in \mathbb{R}^{p} \times \mathbb{R}^{q}: x \geq\|u\| e\right\}
$$


and showed that the dual of $L$ is

$$
L(p, q)^{*}=\left\{(x, u) \in \mathbb{R}^{p} \times \mathbb{R}^{q}:\langle x, e\rangle \geq\|u\|, x \geq 0\right\} .
$$

In the same paper, we showed the following:

- The extended Lorentz cone $L(p, q)$ defined by (4) is a (regular) cone.

- The cone $L(p, q)$ is polyhedral (see Rockafellar [22]) iff $q=1$.

- The cone $L(p, q)$ is subdual, and $L(p, q)$ is self-dual iff $p=1$, that is, when $L(p, q)=L(1, q)$ is the $q+1$-dimensional Lorentz cone.

$-L(p, q)$ is a self-dual polyhedral cone iff $p=q=1$.

In Theorem 2 of Nemeth and Zhang [8], we determined the $L(p, q)$-isotone projection sets. For convenience, we repeat this theorem here:

Theorem 3.1 1. Let $K=\mathbb{R}^{p} \times C$, where $C$ is an arbitrary nonempty, closed, and convex set in $\mathbb{R}^{q}$ and $L(p, q)$ be the extended Lorentz cone defined by (4). Then, $K$ is an $L(p, q)$-isotone projection set.

2. Let $p=1, q>1$ and $K \subseteq \mathbb{R}^{p} \times \mathbb{R}^{q}$ be a nonempty, closed, and convex set. Then, $K$ is an $L(p, q)$-isotone projection set iff $K=\mathbb{R}^{p} \times C$, for some $C \subseteq \mathbb{R}^{q}$ nonempty, closed, and convex set.

3. Let $p, q>1$, and

$$
K=\cap_{\ell \in \mathbb{N}} \mathcal{H}_{-}\left(\gamma^{\ell}, \beta^{\ell}\right) \subseteq \mathbb{R}^{p} \times \mathbb{R}^{q},
$$

where $\gamma^{\ell}=\left(a^{\ell}, u^{\ell}\right)$ is a unit vector. Then, $K$ is an $L(p, q)$-isotone projection set iff for each $\ell$ one of the following conditions hold:

(a) The vector $a^{\ell}=0$.

(b) The vector $u^{\ell}=0$, and there exists $i \neq j$ such that $a_{i}^{\ell}=\sqrt{2} / 2$, $a_{j}^{\ell}=-\sqrt{2} / 2$ and $a_{k}^{\ell}=0$, for any $k \notin\{i, j\}$.

\section{Variational Inequalities}

Let $K \subseteq \mathbb{R}^{m}$ be a closed and convex set and $F: \mathbb{R}^{m} \rightarrow \mathbb{R}^{m}$ be a mapping. Then, the variational inequality $V I(K, F)$ defined by $F$ and $K$ is the problem of finding an $x \in K$ such that for any $y \in K$,

$$
\langle y-x, F(x)\rangle \geq 0 .
$$

It is known that $x^{*}$ is a solution of $\operatorname{VI}(F, K)$ if and only if it is a fixed point of the mapping $P_{K} \circ(I-F)$, where $I$ is the identity mapping of $\mathbb{R}^{m}$ (see Facchinei and Pang [1]). Consider the Picard iteration

$$
x^{n+1}=P_{K}\left(x^{n}-F\left(x^{n}\right)\right) .
$$

If $F$ is continuous and $\left\{x^{n}\right\}_{n \in \mathbb{N}}$ is convergent to $x^{*}$, then it follows that $x^{*}$ is a fixed point of the mapping $P_{K} \circ(I-F)$ and hence a solution of $V I(F, K)$. Therefore, it is natural to seek convergence conditions for $x^{n}$. Let us first state the following simple lemma: 
Lemma 4.1 Let $K \subseteq \mathbb{R}^{m}$ be a closed and convex set, $F: \mathbb{R}^{m} \rightarrow \mathbb{R}^{m}$ be a continuous mapping, and L be a cone. Consider the sequence $\left\{x^{n}\right\}_{n \in \mathbb{N}}$ defined by (5). Suppose that the mappings $P_{K}$ and $I-F$ are L-isotone, $x^{0} \leq_{L} x^{1}$, and there exists a $y \in \mathbb{R}^{m}$ such that $x^{n} \leq_{L} y$, for all $n \in \mathbb{N}$ sufficiently large. Then, $\left\{x^{n}\right\}_{n \in \mathbb{N}}$ is convergent, and its limit $x^{*}$ is a solution of $V I(F, K)$.

Proof Since the mappings $P_{K}$ and $I-F$ are $L$-isotone, the mapping $x \mapsto P_{K} \circ(I-F)$ is also $L$-isotone. Then, by using (5) and a simple inductive argument, it follows that $\left\{x^{n}\right\}_{n \in \mathbb{N}}$ is $L$-increasing. Since any cone in $\mathbb{R}^{m}$ is regular, $\left\{x^{n}\right\}_{n \in \mathbb{N}}$ is convergent, and hence, its limit $x^{*}$ is fixed point of $P_{K} \circ(I-F)$ and therefore a solution of $V I(F, K)$.

Remark 4.1 Consider the assumptions of Lemma 4.1. If we further suppose that $I-F$ is nonexpansive, then $P_{K} \circ(I-F)$ is also nonexpansive. Hence, the limit in Lemma 4.1 is robust in the sense that if the starting points $x^{0}$ and $y^{0}$ are close to each other, then the corresponding limits $x^{*}$ and $y^{*}$ are also closed to each other.

Remark 4.2 The condition $x^{0} \quad \leq_{L} x^{1}$ in Lemma 4.1 is satisfied when $x^{0} \in K \cap F^{-1}(-L)$. Indeed, if $x^{0} \in K \cap F^{-1}(-L)$, then $-F\left(x^{0}\right) \in L$ and $x^{0} \in K$. Thus, $x^{0} \leq_{L} x^{0}-F\left(x^{0}\right)$, and hence, by the isotonicity of $P_{K}$, we obtain $x^{0}=P_{K}\left(x^{0}\right) \leq_{L} P_{K}\left(x^{0}-F\left(x^{0}\right)\right)=x^{1}$.

Proposition 4.1 Let $K \subseteq \mathbb{R}^{m}$ be a closed and convex set, $F: \mathbb{R}^{m} \rightarrow \mathbb{R}^{m}$ be a continuous mapping, and $L$ be a cone. Consider the sequence $\left\{x^{n}\right\}_{n \in \mathbb{N}}$ defined by (5). Suppose that the mappings $P_{K}$ and $I-F$ are L-isotone and $x^{0} \leq_{L} x^{1}$. Denote by $I$ the identity mapping. Let

$$
\begin{aligned}
& \Omega=\left\{x \in K \cap\left(x^{0}+L\right): F(x) \in L\right\} \\
& \Gamma=\left\{x \in K \cap\left(x^{0}+L\right): P_{K}(x-F(x)) \leq_{L} x\right\} .
\end{aligned}
$$

Consider the following statements:

(i) $\Omega \neq \varnothing$,

(ii) $\Gamma \neq \varnothing$,

(iii) The sequence $\left\{x^{n}\right\}_{n \in \mathbb{N}}$ is convergent, and its limit $x^{*}$ is a solution of $V I(F, K)$. Moreover, $x^{*}$ is the L-least element of $\Gamma$.

Then, $\Omega \subseteq \Gamma$ and $(i) \Longrightarrow(i i) \Longrightarrow$ (iii).

Proof (i) $\Longrightarrow$ (ii): Let $y \in \Omega$. Since $P_{K}$ is $L$-isotone, $y-F(y) \leq_{L} y$ implies $P_{K}(y-F(y)) \leq_{L} P_{K}(y)=y$, which shows that $y \in \Gamma$. Hence, $\Omega \subseteq \Gamma$. Thus, (i) $\Longrightarrow$ (ii) is trivial now.

(ii) $\Longrightarrow$ (iii):

Suppose that $\Gamma \neq \varnothing$. Since the mappings $P_{K}$ and $I-F$ are $L$-isotone, the mapping $P_{K} \circ(I-F)$ is also $L$-isotone. Similarly to the proof of Lemma 4.1, it can be shown that $\left\{x^{n}\right\}_{n \in \mathbb{N}}$ is $L$-increasing. Let $y \in \Gamma$ be arbitrary but fixed. We have $y-x^{0} \in L$, that is, $x^{0} \leq_{L} y$. Suppose that $x^{n} \leq_{L} y$. We show, by induction, that $x^{n} \leq_{L} y$ for all $n \geq 0$. Since the mapping $P_{K} \circ(I-F)$ is $L$-isotone, $x^{n} \leq_{L} y$ implies that

$$
x^{n+1}=P_{K}\left(x^{n}-F\left(x^{n}\right)\right) \leq_{L} P_{K}(y-F(y)) \leq_{L} y .
$$


Thus, $x^{n} \leq_{L} y$ for all $n \geq 0$. Then, Lemma 4.1 implies that $\left\{x^{n}\right\}_{n \in \mathbb{N}}$ is convergent, and its limit $x^{*} \in K \cap\left(x^{0}+L\right)$ is a solution of $V I(F, K)$. Since $x^{*}$ is a solution of $V I(F, K)$, we have that $P_{K}\left(x^{*}-F\left(x^{*}\right)\right)=x^{*}$ and hence $x^{*} \in \Gamma$. Moreover, the relation $x^{n} \leq_{L} y$ in limit gives $x^{*} \leq y$. Therefore, $x^{*}$ is the smallest element of $\Gamma$ with respect to the partial order defined by $L$.

\section{Variational Inequalities on Cylinders}

Let $p, q$ be positive integers and $m=p+q$. By a cylinder, we mean a set $K=$ $\mathbb{R}^{p} \times C \subseteq \mathbb{R}^{p} \times \mathbb{R}^{q} \equiv \mathbb{R}^{m}$, where $C$ is a nonempty, closed and convex subset of $\mathbb{R}^{q}$. In this section, we will specialize the results of the previous section for variational inequalities on cylinders.

Lemma 5.1 Let $K=\mathbb{R}^{p} \times C$, where $C$ is an arbitrary nonempty, closed, and convex set in $\mathbb{R}^{q}$. Let $G: \mathbb{R}^{p} \times \mathbb{R}^{q} \rightarrow \mathbb{R}^{p}, H: \mathbb{R}^{p} \times \mathbb{R}^{q} \rightarrow \mathbb{R}^{q}$ and

$$
F=(G, H): \mathbb{R}^{p} \times \mathbb{R}^{q} \rightarrow \mathbb{R}^{p} \times \mathbb{R}^{q}
$$

Then, the variational inequality $V I(F, K)$ is equivalent to the problem of finding a vector $(x, u) \in \mathbb{R}^{p} \times C$ such that

$$
G(x, u)=0,\langle v-u, H(x, u)\rangle \geq 0, \forall v \in C .
$$

Proof The variational inequality $V I(F, K)$ is equivalent to finding an $(x, u) \in \mathbb{R}^{p} \times C$ such that

$$
\langle y-x, G(x, u)\rangle+\langle v-u, H(x, u)\rangle \geq 0, \forall(y, v) \in \mathbb{R}^{p} \times C .
$$

Let $(x, u) \in \mathbb{R}^{p} \times C$ be a solution of (7). If we choose $v=u \in C$ in (7), then we get $\langle y-x, G(x, u)\rangle \geq 0$ for any $y \in \mathbb{R}^{p}$. Hence, $G(x, u)=0$ and $\langle v-u, H(x, u)\rangle \geq 0$. Conversely, if $(x, u) \in \mathbb{R}^{p} \times C$ is a solution of (6), then it is easy to see that it is a solution of (7).

By using the notation of Lemma 5.1, the Picard iteration (5) can be rewritten as:

$$
\begin{aligned}
& x^{n+1}=x^{n}-G\left(x^{n}, u^{n}\right), \\
& u^{n+1}=P_{C}\left(u^{n}-H\left(x^{n}, u^{n}\right)\right) .
\end{aligned}
$$

Consider the partial order defined by the extended Lorentz cone (4). Then, we obtain the following proposition.

Theorem 5.1 Let $K=\mathbb{R}^{p} \times C$, where $C$ is a nonempty, closed, and convex subset of $\mathbb{R}^{q}$. Let $G: \mathbb{R}^{p} \times \mathbb{R}^{q} \rightarrow \mathbb{R}^{p}, H: \mathbb{R}^{p} \times \mathbb{R}^{q} \rightarrow \mathbb{R}^{q}$ be continuous mappings, $F=(G, H): \mathbb{R}^{p} \times \mathbb{R}^{q} \rightarrow \mathbb{R}^{p} \times \mathbb{R}^{q}$. Let $\left(x^{0}, u^{0}\right) \in \mathbb{R}^{p} \times \mathbb{R}^{q}$ and consider the sequence $\left(x^{n}, u^{n}\right)_{n \in \mathbb{N}}$ defined by (8). Let $x, y \in \mathbb{R}^{p}$ and $u, v \in \mathbb{R}^{q}$. Suppose that 
$x^{1}-x^{0} \geq\left\|u^{1}-u^{0}\right\| e$ (in particular, by Remark 4.2, this holds if $u^{0} \in C$ and $\left.-G\left(x^{0}, u^{0}\right) \geq\left\|H\left(x^{0}, u^{0}\right)\right\| e\right)$ and that $y-x \geq\|v-u\| e$ implies

$$
y-x-G(y, v)+G(x, u) \geq\|v-u-H(y, v)+H(x, u)\| e .
$$

Let

$$
\Omega=\left\{(x, u) \in \mathbb{R}^{p} \times C: x-x^{0} \geq\left\|u-u^{0}\right\| e, G(x, u) \geq\|H(x, u)\| e\right\}
$$

and

$$
\begin{aligned}
& \Gamma=\left\{(x, u) \in \mathbb{R}^{p} \times C:\right. x-x^{0} \geq\left\|u-u^{0}\right\| e, \\
&\left.G(x, u) \geq\left\|u-P_{C}(u-H(x, u))\right\| e\right\} .
\end{aligned}
$$

Consider the following statements:

(i) $\Omega \neq \varnothing$,

(ii) $\Gamma \neq \varnothing$,

(iii) The sequence $\left\{\left(x^{n}, u^{n}\right)\right\}_{n \in \mathbb{N}}$ is convergent, and its limit $\left(x^{*}, u^{*}\right)$ is a solution of $V I(F, K)$. Moreover, $\left(x^{*}, u^{*}\right)$ is the smallest element of $\Gamma$ with respect to the partial order defined by the extended Lorentz cone $L(p, q)$ defined by (4).

Then, $\Omega \subseteq \Gamma$ and $(i) \Longrightarrow($ ii $) \Longrightarrow($ iii).

Proof Let $L(p, q)$ be the extended Lorentz cone defined by (4). First, observe that $K \cap\left(\left(x^{0}, u^{0}\right)+L(p, q)\right) \neq \varnothing$. By using the definition of the extended Lorentz cone, it is easy to verify that

$$
\begin{aligned}
\Omega & =K \cap\left(\left(x^{0}, u^{0}\right)+L(p, q)\right) \cap F^{-1}(L(p, q)) \\
& =\left\{z \in K \cap\left(\left(x^{0}, u^{0}\right)+L(p, q)\right): F(z) \in L(p, q)\right\}
\end{aligned}
$$

and

$$
\Gamma=\left\{z \in K \cap\left(\left(x^{0}, u^{0}\right)+L(p, q)\right): P_{K}(z-F(z)) \leq_{L(p, q)} z\right\} .
$$

Let $x, y \in \mathbb{R}^{p}$ and $u, v \in C$. Since $y-x \geq\|v-u\| e$ implies

$$
y-x-G(y, v)+G(x, u) \geq\|v-u-H(y, v)+H(x, u)\| e,
$$

it follows that $I-F$ is $L(p, q)$-isotone. Hence, by Proposition 4.1 (with $m=p+q$ ), Thorem 3.1 and Lemma 5.1, it follows that $\Omega \subseteq \Gamma$ and (i) $\Longrightarrow$ (ii) $\Longrightarrow$ (iii). 


\section{Affine Variational Inequalities on Cylinders}

Throughout this section, we will use the notation of Proposition 4.1, and we will suppose that $\operatorname{int}(L)$ is nonempty and $P_{K}$ is $L$-isotone, which is true for the extended Lorentz cone $L=L(p, q)$. We will present a large class of monotone solvable affine variational inequalities for which (8) is monotone and convergent.

\section{Lemma 6.1 If}

1. The mapping $I-F$ is L-isotone, $x^{0} \in K$ and $F\left(x^{0}\right) \in-L$ and if there exists an $x \in \mathbb{R}^{m}$ such that

2. We have the inclusions $x \in K, F(x) \in L$ and $x-x^{0} \in L$, then (5) ((8) if $L=L(p, q))$ is convergent.

Proof By Remarks 4.1 and 4.2, Condition 1 of the lemma implies $x^{0} \leq_{L} x^{1}$, and Condition 2 of the lemma means that $x \in \Omega$, that is, $\Omega$ is nonempty. Hence, the result follows from Proposition 4.1.

For any $m \times m$ matrix $M$ and set $\Lambda \subseteq \mathbb{R}^{m}$ denote by int $\Lambda$ the interior of $\Lambda$ and by $\|M\|$ the operator norm of $M$, i.e.,

$$
\|M\|=\min \left\{c \geq 0:\|M x\| \leq c\|x\| \text { for all } x \in \mathbb{R}^{m}\right\}
$$

and let $M \Lambda:=\{M x: x \in \Lambda\}$.

Lemma 6.2 Suppose that $F$ is an affine mapping, that is, $F(z)=A z+b, \forall z \in \mathbb{R}^{m}$, where $A$ is a constant $m \times m$ nonsingular matrix and $b \in \mathbb{R}^{m}$ is a constant vector. Let $x^{0} \in A^{-1}(-b-L)$. If $(I-A) L \subseteq L$ and $A L \cap \operatorname{int}(L) \neq \varnothing$, then there exists an $x \in \mathbb{R}^{m}$ such that $F(x) \in L$ and $x-\bar{x}^{0} \in L$ and if $K$ is a closed and convex set such that $x, x^{0} \in K$, then (5) ((8) if $\left.L=L(p, q)\right)$ is convergent.

Proof Note that $x^{0} \in A^{-1}(-b-L)$ means that $F\left(x^{0}\right) \in-L$ and $(I-A) L \subseteq L$ is equivalent to $I-F$ is $L$-isotone (as remarked by one of the reviewers, in case of $L=\mathbb{R}_{+}^{m}$, this implies that $A$ has the $Z$-property, that is, it has nonnegative off-diagonal entries). Let

$$
A y \in A L \cap \operatorname{int}(L) .
$$

Then, $y \in L$ and $A y \in \operatorname{int}(L)$. Hence, there exists a sufficiently large positive real number $\lambda$ such that $(1 / \lambda)\left(A x^{0}+b\right)+A y \in L$. Then, $A x+b \in L$, where $x=x^{0}+\lambda y$. Hence, $F(x) \in L$ and $x-x^{0} \in L$. Choose $K$ to be a closed and convex set such that $K$ contains $x^{0}$ and $x$ (for example, in case of the box-constrained variational inequalities of the next section, choose the box large enough to contain $x^{0}$ and $x$ ). Then, Conditions 1-2 of Lemma 6.1 are satisfied, and therefore, (5) ((8) if $L=L(p, q))$ is convergent. 
In conclusion, satisfying Conditions 1-2 of Lemma 6.1 reduces to finding nonsingular matrices $A$ such that $(I-A) L \subseteq L$ and $A L \cap \operatorname{int}(L) \neq \varnothing$. Let us concentrate on the latter problem for $L=L(p, q)$, the extended Lorentz cone, when the conditions of Proposition 4.1 become the conditions of Theorem 5.1.

Usually, $I_{r}$ denotes the identity matrix in $\mathbb{R}^{r}$. However, in our case, the notation $I$ will always be unambiguous, and therefore, we omit the index $r$.

Proposition 6.1 Let $\alpha \in] 0,1[$ be a real constant, $S$ be a $p \times p$ constant matrix with all entries in the main diagonal from the interval $] \alpha, 1[$ and the sum of the elements in each of its row less than $1, T$ be a $q \times q$ matrix such that $\|T\| \leq \alpha$, and $A$ be the block diagonal matrix given by $A:=\left(\begin{array}{cc}I-S & 0 \\ 0 & I-T\end{array}\right)$. Then, $(I-A) L(p, q) \subseteq L(p, q)$ and $A L(p, q) \cap \operatorname{int}(L(p, q)) \neq \varnothing$.

Proof For any $(x, u) \in \mathbb{R}^{p} \times \mathbb{R}^{q}$, we have

$$
A(x, u)=((I-S) x,(I-T) u)
$$

Hence, $(x, u) \in L(p, q)$ is equivalent to $x \geq\|u\| e$, and $A(x, u) \in \operatorname{int}(L(p, q))$ is equivalent to $(I-S) x>\|(I-T) u\| e$. From the restrictions imposed on $S$, it follows that both inequalities will be satisfied if the components of $x$ are equal and large enough. Hence, $(x, u) \in \operatorname{int}(L(p, q)) \neq \varnothing$ and therefore $A L(p, q) \cap \operatorname{int}(L(p, q)) \neq \varnothing$. We have $I-A=\left(\begin{array}{cc}S & 0 \\ 0 & T\end{array}\right)$ and thus

$$
(I-A)(x, u)=(S x, T u)
$$

for any $(x, u) \in L(p, q)$ (i.e., $x \geq\|u\| e$ which implies $x \geq 0)$. Since $S-\alpha I$ is a positive matrix and $x \geq 0$, we get

$$
S x \geq \alpha x \geq\|T\| x \geq\|T\|\|u\| e \geq\|T u\| e .
$$

Therefore, $(I-A)(x, u)=(S x, T u) \in L(p, q)$, which shows that

$$
(I-A) L(p, q) \subseteq L(p, q)
$$

Remark 6.1 Going back to a general cone $L$ with nonempty interior, in the literature the matrices $I-A$ for which $(I-A) L \subseteq L$ are called $L$-positive and form the cone $P(L)$. It is known that $P(L)$ has a nonempty interior as well (see Lemma 5 of Schneider and Vidyasagar [23]). Hence, the inclusion $(I-A) L(p, q) \subseteq L(p, q)$ also holds for some open set in any neighborhood of the matrices of the type $\left(\begin{array}{ccc}I-S & 0 \\ 0 & I-T\end{array}\right)$ constructed above. By continuity reasons, this open set can be chosen so that to satisfy $A L(p, q) \cap \operatorname{int}(L(p, q)) \neq \varnothing$ as well. We conclude that the set of affine mappings $F$ satisfying the condition of Theorem 5.1 is large. 


\section{Unbounded Box-Constrained Variational Inequalities}

Let $p, q$ be positive integers, $m=p+q$ and $K=\prod_{\ell=1}^{m}\left[a_{\ell}, b_{\ell}\right]$ be a box, where $a_{\ell}, b_{\ell} \in \mathbb{R} \cup\{-\infty, \infty\}$ and $a_{\ell}<b_{\ell}$, for all $\ell \in\{1, \ldots, m\}$. The $i$-th entry of the projection mapping is (see, for example, Facchinei and Pang [1]):

$$
\left(P_{K}(x)\right)_{i}=P_{\left[a_{i}, b_{i}\right]}\left(x_{i}\right)=\operatorname{mid}\left(a_{i}, b_{i}, x_{i}\right)=\left\{\begin{array}{l}
a_{i}, \text { if } x_{i} \leq a_{i}, \\
x_{i}, \text { if } a_{i} \leq x_{i} \leq b_{i}, \\
b_{i}, \text { if } b_{i} \leq x_{i}
\end{array}\right.
$$

Let $B=\prod_{i=1}^{p}\left[a_{i}, b_{i}\right] \subseteq \mathbb{R}^{p}$ and $C=\prod_{j=1}^{q}\left[a_{p+j}, b_{p+j}\right]$, so we have

$$
P_{K}(y, v)=\left(P_{B}(y), P_{C}(v)\right)
$$

and the Picard iteration (5) becomes

$$
x_{i}^{n+1}=\operatorname{mid}\left(a_{i}, b_{i},\left(x^{n}-F\left(x^{n}\right)\right)_{i}\right) .
$$

Let $L(p, q)$ be the extended Lorentz cone in $\mathbb{R}^{p} \times \mathbb{R}^{q}$. The next proposition shows that the $L(p, q)$-isotonicity of a box is equivalent to the box being a cylinder. It can be proved either directly, or by using Theorem 2 of Nemeth and Zhang [8]. The details are left to the reader.

Proposition 7.1 Let $L(p, q)$ be the extended Lorentz cone. Then, the projection mapping $P_{K}$ is $L(p, q)$-isotone iff $K=\mathbb{R}^{p} \times C$ where $C=\prod_{j=1}^{q}\left[a_{p+j}, b_{p+j}\right]$.

Hence, the results of Theorem 5.1 can be specialized to the set $K$ given by Proposition 7.1, with the Picard iteration taking the form (11) and the function $\operatorname{mid}\left(a_{i}, b_{i}, \cdot\right)$ given by (9).

\section{Conclusions}

In this paper, we presented a Picard iteration for solving a variational inequality on a cylinder via a fixed point formulation. The iteration is monotonically convergent to the solution of the variational inequality with respect to the partial order defined by an extended Lorentz cone. The monotone convergence is based on the isotonicity of the projection onto a cylinder with respect to the partial order defined by the extended Lorentz cone. We list the following open questions:

1. Given a cone, determine all closed and convex sets onto which the projection is isotone with respect to the partial order defined by the cone.

2. Given a closed and convex set, determine all cones such that the projection onto the closed and convex set is isotone with to respect the partial order defined by the cone.

3. Determine the closed and convex sets for which there exists a cone, such that the projection onto the closed and convex set is isotone with respect to the partial order defined by the cone. 
Although the above questions are difficult to answer in general, any particular result about them can be important for solving complementarity problems and/or variational inequalities by using a monotone convergence. Moreover, any such result could be important in statistics as well, where the isotonicity of the projection may occur in various algorithms (see, for example, the algorithms considered in Guyader, Jegou, Németh and Németh [24]). Some partial results related to Questions 1, 2, and 3 above can be found in Németh and Németh $[25,26]$ and in this paper, but there is still much to be done.

The positive operators on the Lorentz cone have been completely classified in Loewy and Schneider [27]. This classification suggests that the class of affine mappings satisfying Theorem 5.1 is even much larger than the one presented in Sect. 6. However the complete classification of the affine mappings which satisfy the conditions of Theorem 5.1 is still an open question.

Acknowledgments The authors are grateful to the anonymous referees for the many helpful comments and suggestions which greatly contributed to the improvement of this paper.

Open Access This article is distributed under the terms of the Creative Commons Attribution 4.0 International License (http://creativecommons.org/licenses/by/4.0/), which permits unrestricted use, distribution, and reproduction in any medium, provided you give appropriate credit to the original author(s) and the source, provide a link to the Creative Commons license, and indicate if changes were made.

\section{References}

1. Facchinei, F., Pang, J.S.: Finite-Dimensional Variational Inequalities and Complementarity Problems. Springer Series in Operations Research, vol. I. Springer, New York (2003)

2. Isac, G., Németh, A.B.: Isotone projection cones in Hilbert spaces and the complementarity problem. Boll. Unione Mat. Ital. B. 7(4), 773-802 (1990)

3. Isac, G., Németh, A.B.: Projection methods, isotone projection cones, and the complementarity problem. J. Math. Anal. Appl. 153(1), 258-275 (1990)

4. Németh, S.Z.: Iterative methods for nonlinear complementarity problems on isotone projection cones. J. Math. Anal. Appl. 350(1), 340-347 (2009)

5. Abbas, M., Németh, S.Z.: Solving nonlinear complementarity problems by isotonicity of the metric projection. J. Math. Anal. Appl. 386(2), 882-893 (2011)

6. Abbas, M., Németh, S.Z.: Finding solutions of implicit complementarity problems by isotonicity of the metric projection. Nonlinear Anal. 75(4), 2349-2361 (2012). doi:10.1016/j.na.2011.10.033

7. Abbas, M., Németh, S.Z.: Implicit complementarity problems on isotone projection cones. Optimization 61(6), 765-778 (2012). doi:10.1080/02331934.2011.641019

8. Németh, S.Z., Zhang, G.: Extended lorentz cones and mixed complementarity problems. J. Global Optim. 62(3), 443-457 (2015)

9. Auslander, A.: Optimization Méthodes Numériques. Masson, Paris (1976)

10. Bertsekas, D.P., Tsitsiklis, J.N.: Parallel and Distributed Computation: Numerical Methods. PrenticeHall Inc, Englewood Cliffs, New Jersey (1989)

11. Iusem, A.N., Svaiter, B.F.: A variant of Korpelevich's method for variational inequalities with a new search strategy. Optimization 42(4), 309-321 (1997)

12. Khobotov, E.N.: A modification of the extragradient method for solving variational inequalities and some optimization problems. Zhurnal Vychislitel'noi Matematiki i Matematicheskoi Fiziki 27(10), 1462-1473 (1987)

13. Korpelevich, G.M.: The extragradient method for finding saddle points and other problems. Matecon 12, 747-756 (1976)

14. Marcotte, P.: Application of Khobotov's algorithm to variational inequalities and network equilibrium problems. Inf. Syst. Oper. Res. 29, 258-270 (1991) 
15. Nagurney, A.: Network Economics-A Variational Inequality Approach. Kluwer Academic Publishers, Dordrecht, The Netherlands (1993)

16. Sibony, M.: Méthodes itératives pour les équations et inéquations aux dérivées partielles non linéaires de type monotone. Calcolo 7, 65-183 (1970)

17. Solodov, M.V., Svaiter, B.F.: A new projection method for variational inequality problems. SIAM J. Control Optim. 37(3), 765-776 (1999)

18. Solodov, M.V., Tseng, P.: Modified projection-type methods for monotone variational inequalities. SIAM J. Control Optim. 34(5), 1814-1830 (1996)

19. Sun, D.: A class of iterative methods for nonlinear projection equations. J. Optim. Theory Appl. 91(1), 123-140 (1996)

20. Zarantonello, E.: Projections on convex sets in Hilbert space and spectral theory, I: Projections on convex sets, II: Spectral theory. Contrib. Nonlin. Functional Analysis, Proc. Sympos. Univ. Wisconsin, Madison pp. 237-424 (1971)

21. McArthur, C.W.: In what spaces is every closed normal cone regular? Proc. Edinburgh Math. Soc. (2) 17, 121-125 (1970/1971)

22. Rockafellar, R.T.: Convex Analysis. Princeton Univ. Press, Princeton (1970)

23. Schneider, H., Vidyasagar, M.: Cross-positive matrices. SIAM J. Numer. Anal. 7, 508-519 (1970)

24. Guyader, A., Jégou, N., Németh, A.B., Németh, S.Z.: A geometrical approach to iterative isotone regression. Appl. Math. Comput. 227, 359-369 (2014)

25. Németh, A.B., Németh, S.Z.: Lattice-like operations and isotone projection sets. Linear Algebra Appl. 439(10), 2815-2828 (2013)

26. Németh, A.B., Németh, S.Z.: Lattice-like subsets of Euclidean Jordan algebras (2014). arXiv: 1401.3581

27. Loewy, R., Schneider, H.: Positive operators on the $n$-dimensional ice cream cone. J. Math. Anal. Appl. 49, 375-392 (1975) 